CONF-911106--8

DE92 002809

Instrumentation and Controls Division

\title{
DATA ACQUISITION WORKSTATION FOR THE OAK RIDGE ELECTRON LINEAR ACCELERATOR
}

\author{
J. H. Todd \\ B. D. Rooney ${ }^{\dagger}$ \\ R. R. Spencer ${ }^{\dagger}$ \\ L. W. Weston ${ }^{\dagger}$

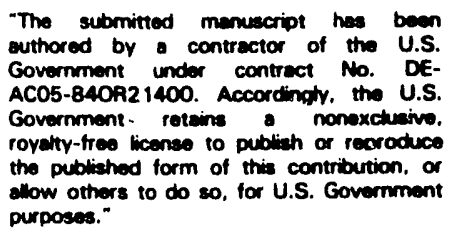 \\ Paper to be presented at the \\ 1991 IEEE Nuclear Science Symposium \\ Santa Fe, New Mexico \\ November 2-9, 1991
}

\section{DISCLAIMER}

This report was prepared as an account of work sponsored by an agency of the United States Government. Neither the United States Government nor any agency thereof, nor any of their Goves any warranty, express or implied, or assumes any legal liability or responsiemployees, makes any warranty, express or implies, of any information, apparatus, product, or bility for the accuracy, completeness, or usefulness of any inge privately owned rights. Referprocess disclosed, or represents that its use would not infrese or service by trade name, trademark, ence herein to any specific commercial product, process, or servimply its endorsement, recommanufacturer, or otherwise does not necessarily constint or any agency thereof. The views mendation, or favoring by the United States Government or any ate or reflect those of the and opinions of authors expressed herein do not

*Research Sponsored by the U. S. Department of Energy and performed at Oak Ridge National Laboratory, managed by Martin Marietta Energy Systems, Inc. for the U. S. Department of Energy under Contract No. DE-AC05-84OR21400. 


\title{
DATA ACQUISITION WORKSTATION FOR OAK RIDGE ELECTRON LINEAR ACCELERATOR*
}

\author{
J. H. Todd, B. D. Rooney, R. H. Spencer, L. W. Weston \\ Oak Ridge National Laboratory \\ Instrumentation and Controls Division \\ P. O. Box 2008, Oak Ridge, TN 37831-6006
}

\begin{abstract}
A new multiparameter data acquisition system for the Oak Ridge Electron Linear Accelerator (ORELA) ${ }^{[1]}$ was developed, fabricated and tested. This system uses an IBM PS/2 Model 80-111 personal computer and a data handler with a 2048-word buffer. The buffer can accept data at a rate exceeding one million events per second in bursts of 512 words of 64-bits length. The acquisition system, limited by software, can acquire data from 1,2 , or 3 digitizers; multiplex up to 4 detectors; read and control up to 16 scalers or registers; and output up to $32 \mathrm{DC}$ logic lines that can be used to control external instrumentation. Software was developed for the OS/2 operating system, supporting multiparameter data storage for up to three million channels. Data can be collected in a background mode to make the computer available for other tasks while collecting data. The system also supports multiparameter biasing and can collect, crunch, and store data at rates of 30,000 events per second, each event containing up to 64-bits of information. A technical manual, ORNL/TM11454 , covering the use of the system has been published.
\end{abstract}

\section{INTRODUCTION}

Nuclear spectrometry frequently involves computers and complex analyzer systems to rapidly analyze, sort, and store applicable data. Many systems can be extremely elaborate and costly, depending on the experiment and the type of information desired. For single-parameter events such as digital time measurements or pulse height analysis, an abundance of commercial analyzers are on the market, many at a reasonable price. However, multiparame ter data acquisition systems involving multiple detectors and digitizers usually require a costly and elaborate computer system with extensive memory. The availability of analyzer systems with storage capacity above one million channels is extremely limited and costly.

Requirements at (ORELA) along with the need to replace aging analyzers and computers within current budgets led to

*Research sponsored in part by the U.S. Navy RADIAC Development Program under DOE Interagency Agreement No. 05310531-A1 and in part by the Defense Advanced Research Projects Agency under DOE Interagency Agreement No. 1868-B135-A1. The Cak Ridge National Laboratory is operated by Martin Marietta Energy Systems, Inc. for the U. S. Department of Energy under Contract No. DE-ACOS-84OR21400.

the design and fabrication of a data acquisition system. This system consists of a hardware interface and data acquisition software that uses an IBM PS/2 Model 80-111 personal computer. This system was developed as the primary replacement for and as an upgrade of older computer equipment. The new system can analyze 64-bits of information per event into 4-parameter storage using nonlinear binning and can employ multiparameter discrimination. Having a maximum capacity of three million channels, the system's low cost makes it possible to provide an independent system for each experimenter.

The system can collect, crunch, and store data at rates as high as 30,000 events per second, each event containing up to 64-bits of data. A temporary memory in the hardware, essentially a hardware DMA, allows bursts of data at rates exceeding $1,000,000$ events per second. This memory contains 2048 words of 16 -bit length. If a data rate exceeds the capabilities of the system, the temporary memory will fill and an alarm will be given to indicate that data is being lost.

An IBM PS/2 personal computer was chosen over its contemporaries because of its architecture, compatibility, interrupt priority capability, and multitasking capability. Collection of data can be done with top priority while using the computer for other tasks such as display and data analysis, making real time analysis of data possible in some situations. Software, in the form of a device driver, allows users to easily write specialized programs that have access to data during acquisition. All software was designed to run under the OS $/ 2$ operating system.

The manual, (ORNL/TM-11454), serves as a user' guide for the data acquisition system. This manual provides the user with detailed information on setting up and using the system for a variety of applications including multiparameter data storage, multiparameter biasing, time of flight energy display, and software development. The manual also serves as a guide for the computer code ANALYZER, a general-purpose program that provides real-time display of data, backup file support, timer support, time-of-flight energy calculations, and other functions. Programs can easily be written in either the protected mode or the DOS-compatible mode to interface with the acquisition driver by following the format specified in the manual. 


\section{GENERAL}

A block diagram of a typical data acquisition system at ORELA is shown in Fig. 1. The system consists of an IBM PS/2 Model 80 personal computer attached to several external devices through a data handler. External instrumentation for the initial implementation includes one ORTEC time digitizer, two Nuclear Data ADC's and eight JORWAY scalers. The experimenter has the option to configure the system for specific and fewer digitizers through switches on the front of the data handler. There are also $32 \mathrm{DC}$ output lines that can be used to control external instrumentation.

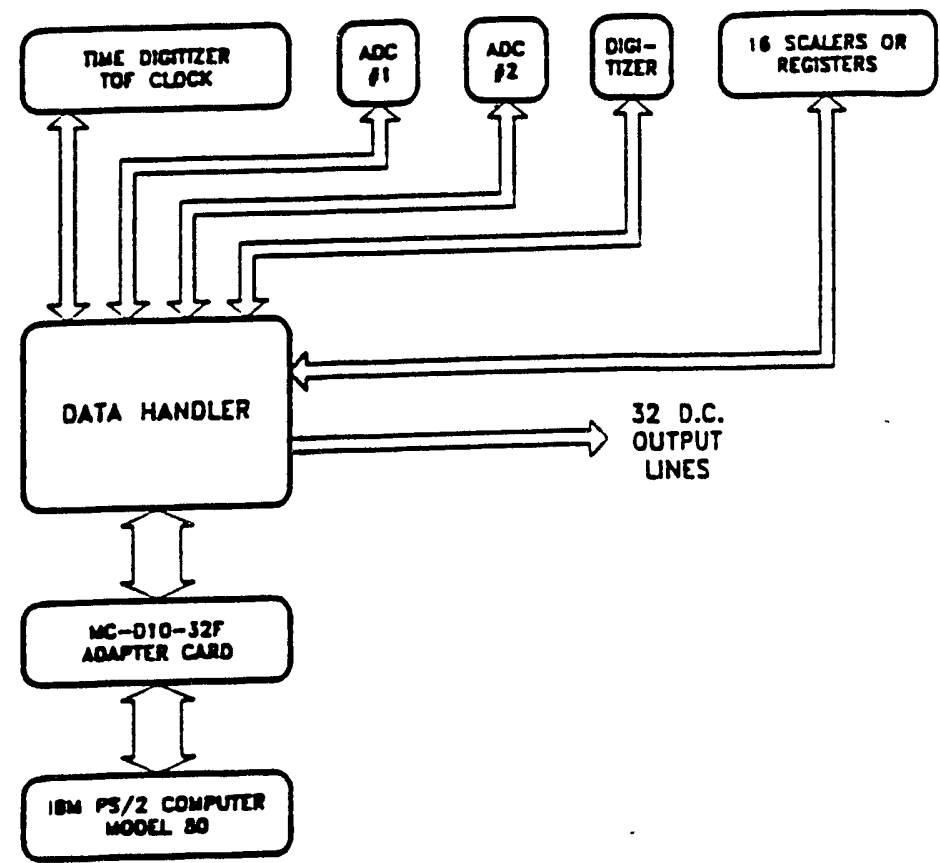

Fig.1 Data Acquisition System Block Diagram

\section{SOFTWARE}

The methodology of software development has been to provide the user with a versatile data acquisition system that can easily interface with any user program in the OS/2 operating system. This provides the user with the ability to expand and enhance data analysis and display routines at his or her leisure and to support possible upgrades in computer hardware.

Three main programs have been written to assist the user in controlling and displaying data; LOADCRUN, ANALYZER, and DEVICE2. Each of these programs are described in detail in Chapters 4, 5, and 6 respectively of ORNL/TM-11454. DEVICE2 is a device driver that controls the data handler, performing all necessary tasks to start, stop, and transfer data. Program LOADCRUN is used to load a crunch file into the device driver. A crunch file is an ASCII data file containing parameters supplied by the user to determine how data is to be binned and stored. ANALYZER provides the user with a general purpose program which starts, stops, and displays data by accessing the device driver. Other data acquisitions programs can easily be written to replace ANALYZER using the format in Chapter 6 and the example in Appendix $\mathrm{C}$ of the manual.

\section{HARDWARE}

Referring again to Fig. 1 as an outline of the data handler integrated into a system, there are 4 ports associated with the data handler as well as one external control line. With the system running the external line is used to start and stop the data taking routine under the control of an external signal. Ports 1 and 2 are data input ports. Port 1 is the 64-bit input from digitizers. Port 2 contains the control lines for 16 scalers or registers and 32 lines for data input. Port 3 is a 32 line output port. Port 4 is an $\mathrm{V} / \mathrm{O}$ port to the $\mathrm{PS} / 2$ computer via the MC-DIO-32F adapter card.

\section{A. Computer}

This data acquisition system utilizes an IBM PS/2 Model 80-111 personal computer to control the data handler and analyze each event. The computer contains an 80386 microprocessor with a $20 \mathrm{Mhz}$ clock and has a 8C0387 math co-processor. Additional adapters installed into the computer consist of an I/O parallel port card and extended memory. Memory may be extended up to 16 megabytes, giving the system approximately three million channel capacity, with each channel consisting of 32-bits (four billion counts per channel). At least 2 megabytes of memory are reserved for the OS/2 operating system.

\section{B. Port Adapter Card}

The computer requires a 32-bit parallel 1/O adapter to enable the computer to communicate with the data handler. The acquisition system has been developed to use a commercially available interface card, MC-DIO-32F, from National Instruments Corporation. This I/O card is installed into one of the computer expansion slots and connected to the data handler using a 50 line ribbon cable.

The MC-DIO-32F adapter must be installed and configured to a base port address of D000 hex before operation. To accomplish this, the user is referred to the instructions that come with the board. The interrupt level and DMA channel are currently not used in this system, by software choice, thus these parameters may be disabled or set to whatever the user desires. All communications between the computer and the system, including data transfer is through this adapter.

\section{Digitizers}

As many as four digitizers may be used in this data acquisition system; however current software supports only three. Which digitizers are employed can be controlled from switches on the front panel of the data handler. The system is currently set up to accept up to 13-bits of data (8192 channels) from two ADC's and 26-bits of data and 4 tags from a time 
digitizer. With the least significant bit at one nanosecond, this gives a range of 1 to 67,108,863 nanoseconds. Additional bits may be used if required; however, this will require some modification in the device driver software. The software has been modified by one experimenter with the addition of a fourth digitizer. The fourth digitizer contains information on the signal source in a multiple detector system (twelve detectors in the system).

\section{Scalers or Registers}

Scaler or register data are $s$ pported by software in this data acquisition system. The data must be presented as a "wired or" with individual gating lines. In addition, there are three BNC connectors on the rear of the data handler that are used to provide start, stop and reset signals to all scalers. On the front panel there are start, stop and reset pushbuttons that are in parallel with the software generated signals.

\section{E. Data Handler}

Figure 2 is a simplified logic diagram of the data handler. The data handler provides the necessary hardware for receiving data from each digitizer and storing it in a $2048 \times 16$-bit word first in first out (FIFO) buffer until the computer is ready to receive it. The FIFO buffer enables the computer to transfer data from the buffer while the data handler is accepting data from the digitizers. The data handler also provides the essential signals required to start, stop, and read up to 16 scalers or registers; however, current software supports only 8 scalers or registers. The device also has an output port with 32 logic lines which can be used to control external parts of the experimental system.

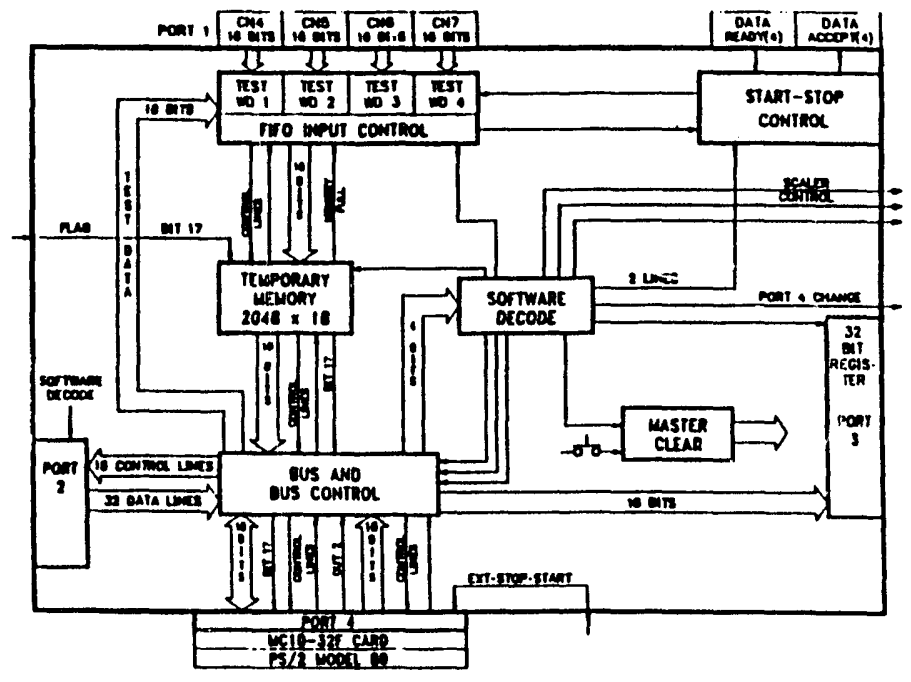

Fig. 2 Data Handler Logic Diagram

\section{E.L. Port 1}

Data is presented to port 1 and can consis: of either 64-bits of data or can consist of 60-bits of data depending upon switch positions on the front panel. The data handler accepts the data as one, two, three or four 16-bit words and stores the data in the temporary memory (FIFO). Additionally, if chosen, a hardware encoder can encode the words with a 1,0,0,0 sequence on the most significant bit to assist in maintaining correlation of the words. This encoding will permit software checks to ensure that correlation of the four words are maintained. If the words are detected out of sequence, action can be initiated by the program. Our software causes a generation of a master clear that removes all data from the FIFO and clears all external data digitizers.

A self test feature is available that inserts the desired number of words per event into the buffer so the computer can compare what is read to what is supposed to be there. The software, upon start up, outputs several words and then reads the words for error comparison. While reading the test input words the software also determines if the data will consist of one, two, three, or four words.

After starting the system, the arrival of a DATA READY signal from the selected number of digitizers initiates the storage of the event and the generation of a DATA ACCEPTED signal that is sent to all external digitizers.

The temporary memory has a capacity of 2048 each 18-bit words. Two of these bits are not used but are available for future uses such as correlation for events having many words.

The input/output of the temporary memory are independent processes. The "FIFO input control" processes the data ready signals from the selected external digitizers. When all of the data ready signals from the selected equipment are present, the data is transferred into memory and a data accepted signal is generated and sent to all external equipment. The time required to accept four 16-bit words has been set for less than one microsecond. The output of data from the temporary memory to the PS/ 2 is controlled by the PS/ 2 through the bus control system. The speed with which data can be removed from the temporary memory via the MO-DIO-32F adapter card varies with the program being used. Measurements have resulted in a transfer rate of approximately $1,000,000$ words of 16-bit length per second. However, software we have used requires approximately 33 microseconds to crunch and store four 16-bit words per event. This corresponds to a rate of about 30,000 64-bit words per second.

\section{E.2. Port 2}

Port 2 is an input port only. This port will accept 32-bits of data. The data must be "wired or" data. There are 16 control or "read" lines that the system will step through to read the data from sixteen scalers or registers.

\section{E. 3. Port 3}

Port 3 is an output port. This port will output DC levels on 32 lines. These 32 lines can be used directly to control equipment or can be decoded to generate up to 232 lines. Timing and duration of signals on the lines are under software 
control. An example of the use of ports 2 and 3 are as follows. Ten of the DC lines from port 3 are used to control filters that are inserted into or removed from a neutron beam. The position of the filters (in or out) are read as a register by port 2 indicating that the desired configuration has actually been achieved. Data collection is permitted upon the correct configuration.

\section{E.4. Pont 4}

Port 4 is attached to the PS $/ 2$ via the MC-DIO-32F adapter card installed into one of the expansion slots in the PS/2. Port 4 contains 32 data lines as well as various control lines. Port 4 is divided into buses 1 and 2. Each bus contains 16bits of bi-directional lines, two handshaking lines, 1 input line and one output line. The input line can be used to set a bit in an internal register that can be monitored by software. This bit is used in the data acquisition system for external control of the start-stop data taking routine. Whenever this bit is low the data taking routine is stopped. The output line reflects the condition of a bit in an internal register that is set or reset by software. This output line is used in the data handler as part of the control system.

The 16 bi-directional lines in bus 1 are used for inputting data into the PS $/ 2$ from the data handler. The one output line and one input line is not used. This line is available for any special use the experimenter desires. Bus 2 is used to output the $32 \mathrm{DC}$ lines and to generate the pulses used for software control upon the output of selected codes. The one output line is used in the data handler to determine if the DC output lines are generated or the software control are generated. As can be seen in Table I codes 0000, Hex 1000, and Hex 1100 are not used and are available for future use. Table I lists the software codes and their functions.

Table I

Software Codes and Functions

\begin{tabular}{|c|c|}
\hline XXXX XXXX XXXX 0000 & Not Used \\
\hline XXXX XXXX XXXX 0001 & Bus to Scalers \\
\hline XXXX XXXX XXXX 0010 & Stop Scalers \\
\hline XXXX XXXX XXXX 0011 & Reset Scalers \\
\hline XXXX XXXX XXXX 0100 & Start Scalers \\
\hline \begin{tabular}{|l|l|}
$X X X X X X X X X X X 101$ \\
\end{tabular} & Bus to Data Input \\
\hline XXXX XXXX XXXX 0110 & Test Data Out \\
\hline \begin{tabular}{|llll}
$X X X X$ & $X X X X$ & $X X X X$ & 0111 \\
\end{tabular} & Master Clear \\
\hline $\begin{array}{lll} & X X X X X X X X X X X X & 1000\end{array}$ & Not Used \\
\hline \begin{tabular}{|l|l|}
$X X X X X X X X X X X 1001$ \\
\end{tabular} & Step Through Scaler Reads \\
\hline 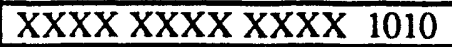 & Start System Acquire \\
\hline 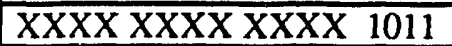 & Stop System Acquire \\
\hline $\mathrm{XXXXXXXXXXXX} 1100$ & Not Used \\
\hline XXXX XXXX XXXX 1101 & Output Test Word \\
\hline $\begin{array}{lll}X X X X X X X X X X & 1110\end{array}$ & $\begin{array}{l}\text { Pulse Indicates DC Line } \\
\text { Change }\end{array}$ \\
\hline $\mathrm{XXXX} \mathrm{XXXX} \mathrm{XXXX} 1111$ & Removes Bus from all $P$ \\
\hline
\end{tabular}

\section{E.5. Software Control}

Control of the data handler is accomplished by the computer through a commercially available I/O board, which allows the computer to communicate with the data handler and transfer information. The data handler has been designed to accept and store data from each digitizer until the computer is ready to analyze it. The scalers are controlled likewise with all data being transferred via the same interface. Transfer and processing of data is performed by the following procedure.

1) The data handler receives a data ready signal from each of the selected digitizers, informing the data handler that there is converted data ready to be transferred.

2) The data handler stores the data from each digitizer into a buffer (FIFQ) and then simultaneously resets each digitizer. This is performed in less than one microsecond after which each digitizer is ready to acquire new data.

3) The CPU checks the most significant bit of each word using a 1,0,0,0 sequence to ensure the correct number of 16-bit words have been transferred for every event.

4) After the correct number of words have been transferred, as determined by the word select switch, the event is analyzed and stored in its applicable channel(s).

5) After the FIFO buffer in the data handler is emptied, the computer returns to the current program or process that was halted.

\section{E.6. Data Rates}

The dead time of the data handler is less than one microsecond. This permits an input rate of $1,000,0000$ events per second for bursts of data not exceeding the buffer capacity of 2048 words. The MC-DIO-32F interface performs all the necessary handshaking requirements with the data handler to place any data in the FIFO buffer directly onto the designated port address in the computer. This transfer to the computer is accomplished in less than 200 nanoseconds, giving programs almost immediate access to data.

The maximum average input rate of this data acquisition system over an extended period of time is highly dependent on the crunch table loaded into memory. For example, the acquisition driver is capable of taking one event and crunching and storing it in up to 9 different locations. The driver can also perform multiparameter discrimination on each event for multiple detectors. All of this takes time. Meàsurements, using software referenced in the manual, have resulted in input rates as fast as 30,000 events per second when using a simple crunch routine and then one store. This was performed while the computer was running sntirely in the protected mode. Acquiring data in the DOS compatibility mode results in a $10 \%$ reduction in the input rate due to the operating system switching in and out of the protected mode during data storage. 
If desired, additional performance and speed may be obtained by modifying the data acquisition driver software. Simplifying the crunch and interrupt routines in the device driver can substantially affect the maximum average input rate. Measurements have resulted in data acquisition rates in excess of 100,000 events per second for simple storage routines; however, modifying the data acquisition driver is only recommended for very specific applications where flexibility is not a requirement.

Use of the IBM Model 80-Al6 computer, with a $25 \mathrm{Mhz}$ clock and 64 Kilobytes of cache memory will greatly increase the data input rate. One data acquisition routine used at ORELA had a six fold increase in the acceptable input data rate with the model 80-A16, with no other change in either the hardware or software.

\section{E.7. Front Panel Switches and Connectors}

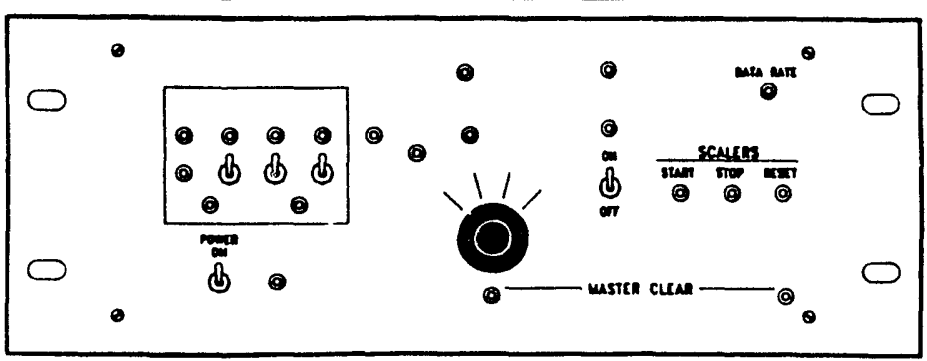

Fig. 3 Front Panel for PS/2 Data Handler

POWER (ON/OFF): This switch provides power to the handler. The power should be be turned off prior or disconnecting any of the rear panel connectors.

DATA READY SWITCHES (ON/OFF): These four switches enable or disable the data ready signals and determine which digitizers must supply a data ready signal before the data handler recognizes, a valid event. Table II illustrates the proper settings for these switches for various combinations of digitizers.

Table II

Front Panel Switch Settings

\begin{tabular}{|l|l|l|l|l|c|}
\hline \multicolumn{7}{|c|}{ DATA READY } \\
\hline & SW1 & SW2 & sW3 & SW4 & $\begin{array}{c}\text { Word } \\
\text { Select }\end{array}$ \\
\hline Digitizer & ON & OFF & OFF & OFF & 2 \\
\hline TOF $^{1}$ Only & ON & OFF & OFF & OFF & 2 \\
\hline PH1 $^{2}$ Only & ON & OFF & OFF & OFF & 2 \\
\hline PH2 ${ }^{3}$ Only & ON & OFF & OFF & OFF & 2 \\
\hline TOF \& PH1 & ON & OFF & OFF & OFF & 2 \\
\hline PH1 \& PH2 & ON & OFF & OFF & OFF & 2 \\
\hline TOF, PH1, \& PH2 & &
\end{tabular}

${ }^{1}$ TOF refers to the clock digitizer

2PH1 refers to pulse height analyzer \#1

${ }^{3} \mathrm{PH} 2$ refers to pulse height analyzer \#2

DATA READY (BNC): These four BNC connectors are attached to the four data ready lines on connectors $\mathrm{Cn} 4$ through
$\mathrm{Cn} 7$ on the rear panel. The connectors therefore provide monitor points.

ALL DATA READY (BNC): This connector provides a monitoring point indicating that the selected data ready signals are present.

ALL DATA READY START (BNC): This connector is a monitor point of the combined data ready signals as seen by the input to the FIFO.

MSB WD ENCODING (IN/OUT); When set to the IN position, this switch sets the most significant bit of each word transferred to memory using a $1,0,0,0$ pattern for up to four words. Software requires that this switch be set to the IN position at all times.

MASTER RESET: The two pushbuttons with this label between them must be depressed simultaneously. This action clears all data from the temporary memory, generates a data accepted signal to the digitizers, and removes the bus from the temporary niemory. The system must be restarted after this action. The master clear can. be generated by software. The software can, of course, restart the system after a program generated master clear.

WORD SELECT (1-4): This switch determines the number of 16-bit words stored for each valid event. Software assumes the first word contain the most significant bits coming from a time digitizer and the second contains the least significant bits. The software also assumes that the other two 16-bit words contain data from two other digitizers. Our software requires that this switch must be set to at least 2 words.

DATA ACCEPTED: (BNC) This BNC is a monitor point for the signal sent to all digitizers after each valid event.

START MONITOR (BNC): This BNC is a monitor point for the signal indicating that the system is in the mode to accept data.

EXT DATA ACCEPT INPUT (BNC); A logic pulse (TTL $\mathrm{HIGH}$ ) input to this connector will produce a data accepted output to all digitizers. This input can be used to ensure correlation of data contained in all external digitizers.

EXT DATA ACCEPT INIPUT ON/OFF (SWITCH): This switch enables/disables the above input. Switch must be in the OFF position if the input is not used.

SCALER START SWITCH: This pushbutton generates a start scaler signal to the rear panel BNC connector. This signal can also be generated by software. This signal will drive 50 ohms with $a+12$ volt pulse.

SCALER STOP SWITCH: Same as the above signal except it is for the STOP function.

SCALE RESET SWITCH: Same as START signal except it is for the reset function. 
ACCEPT DATA (LED): This LED is lighted when the system is in the acquire mode.

DATA RATE HIGH (LED) This LED is lighted when the temporary memory is full. This is an indication that the data rate is high enough to fill the FIFO and that data is being lost.

\section{E.8. Rear Panel Connectors}

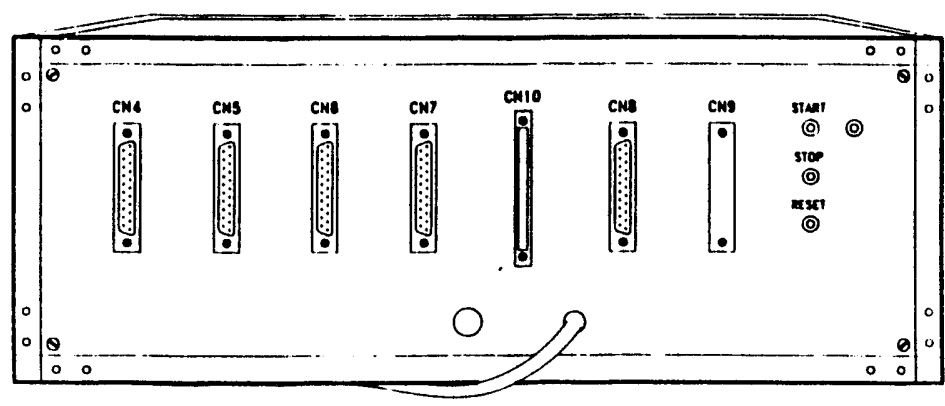

Fig. 4 Rear Panel Connectors for PS/2 Data Handler

CN4 (WORD 1 INPUT): This connector corresponds to the first 16-bit word stored for each valid event. This connector is normally attached to the tags and the most significant bits of the time digitizers. The pin connectors are listed both in the manual and in the drawings of the system.

CN5 (WORD 2 INPUT): This input connector corresponds to the second 16-bit word stored for every valid event. It is normally attached to the less significant bit of the time digitizers. The pin connections are listed in the manual and in the drawings.

CN6 (WORD 3 INPUT): This input connector corresponds to the third word stored. This connector is normally attached to the output of an ADC. The pin connections are listed in the manual and in the drawings.

CN7 WORD 4 INPUT): This corresponds to the fourth word stored. This connector is normally attached to the output of an ADC. The pin connections are listed in the manual and in the drawings.

CN8 (DC LINES OUT): This is a 37-pin connector which supplies the $32 \mathrm{DC}$ out put lines that are available to control external instrumentation. The signals are standard TTL levels. The pin connections are listed in the manual and in the drawings.

CN9 (SCALER INPUT AND CONTROL): This connector contains the 32 input lines and 16 outpu: control lines that are used to read data from external scaless or registers. The pin connections are listed in the manual and in the drawings.

CN10 (Computer): This connector attaches the data handier to the MC-DIO-32F interface card located in one of the computer expansion slots. All data and control signals to and from the computer are routed through this connector.
START STOP AND RESET (BNC): These connectors contain the scaler or register signals as outlined earlier.

DC CHANGE (BNC): This connector contains a pulse that is generated by software whonever a change is made in the DC lines.

\section{SUMMARY}

The Data Acquisition Workstation system provides a flexible and versatile data handling system for a variety of measurements in the nuclear as well as other fields at a reasonable cost.

\section{REFERENCES}

[1] N. C. Pering and T. A. Lewis, IEEE Trans. Nucl. Sci. , 18, 3 , 929. (1971). 

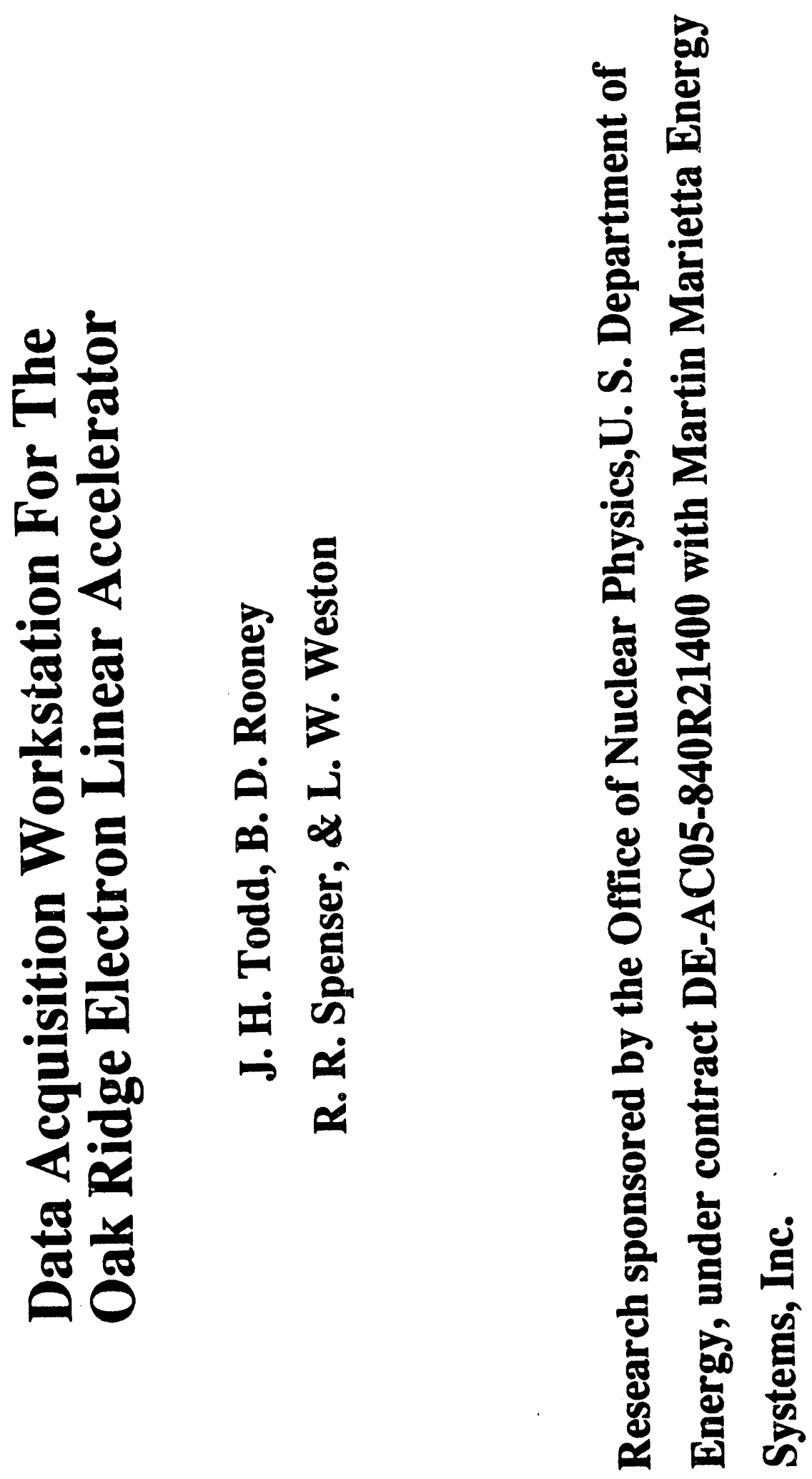


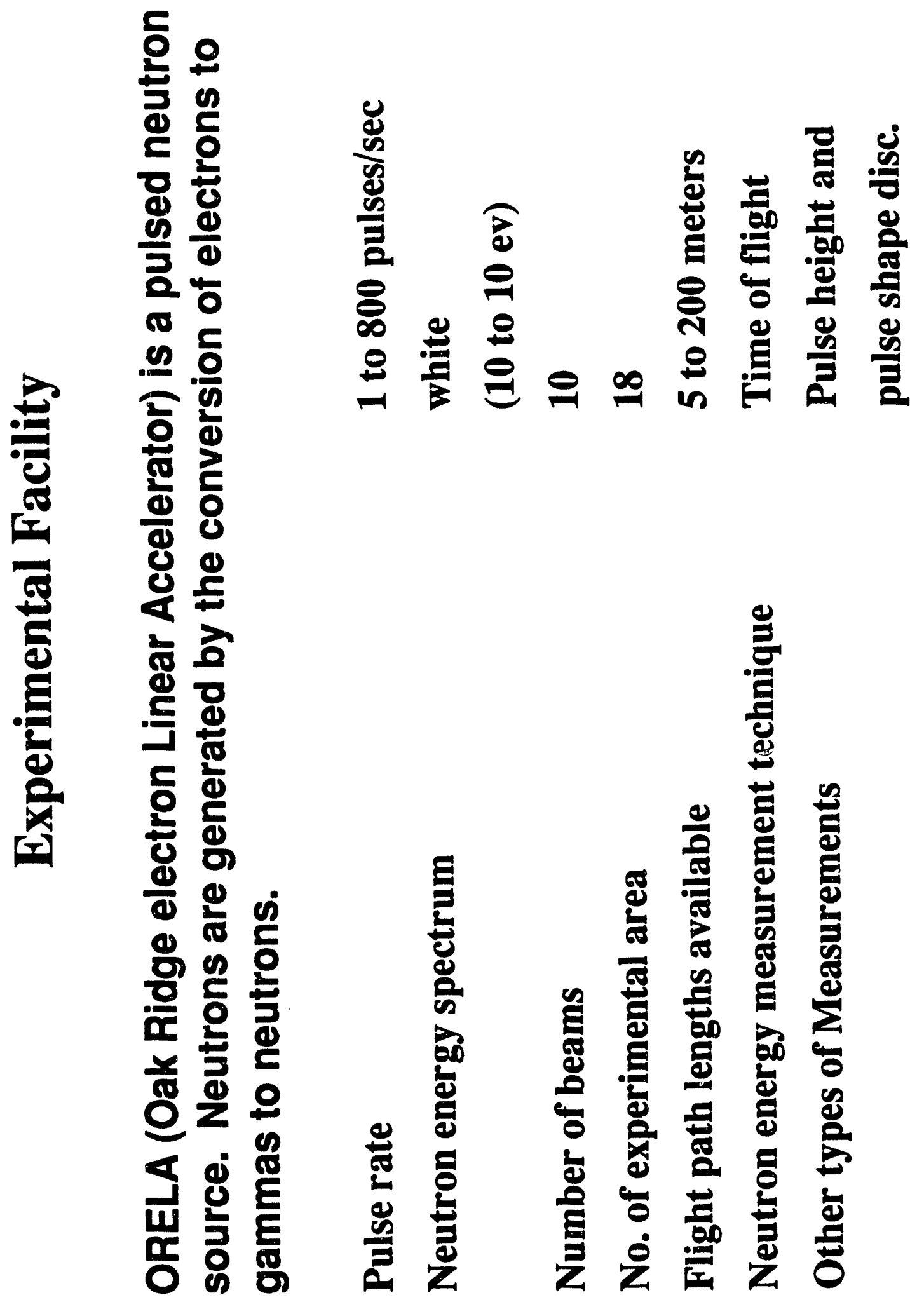




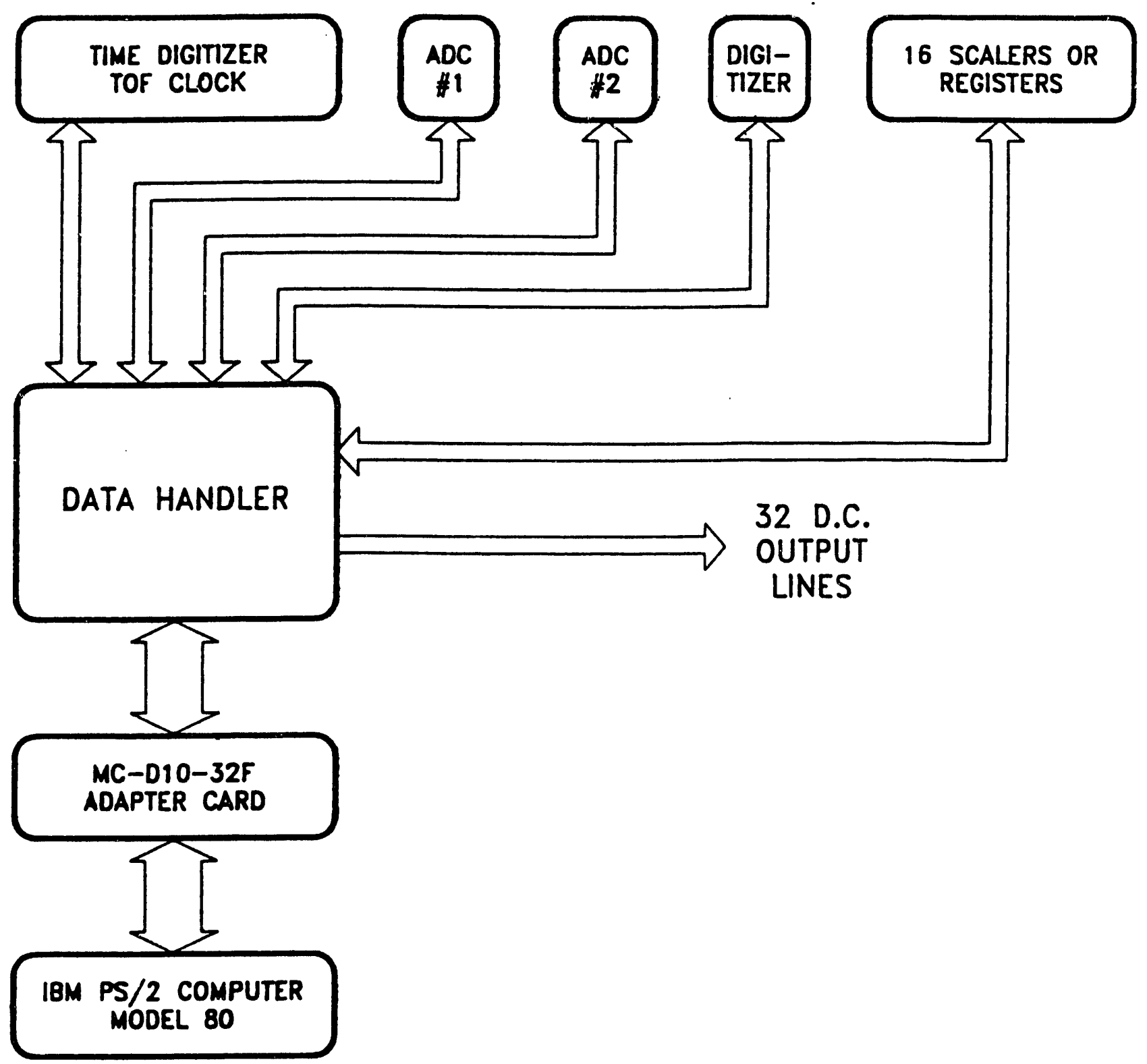



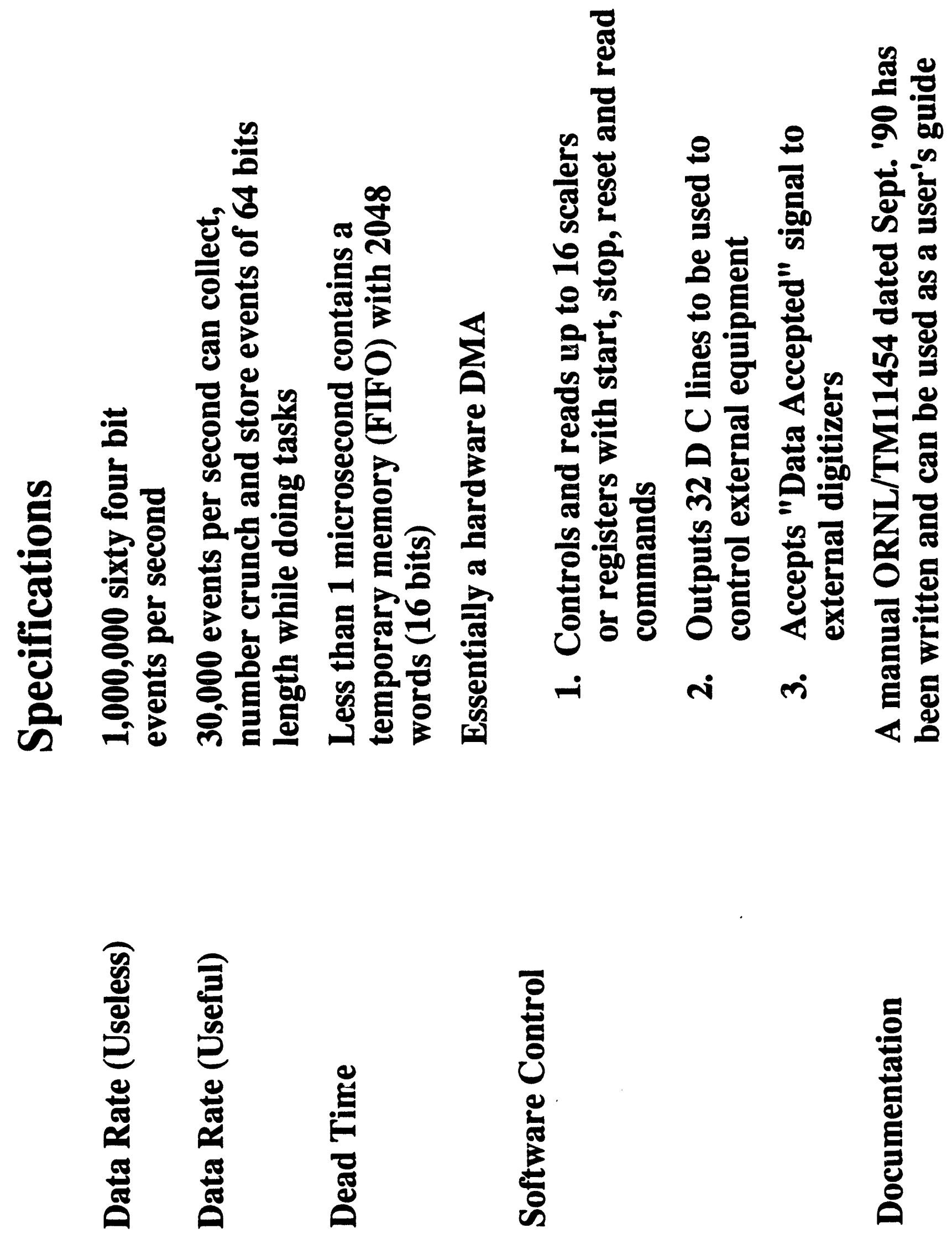


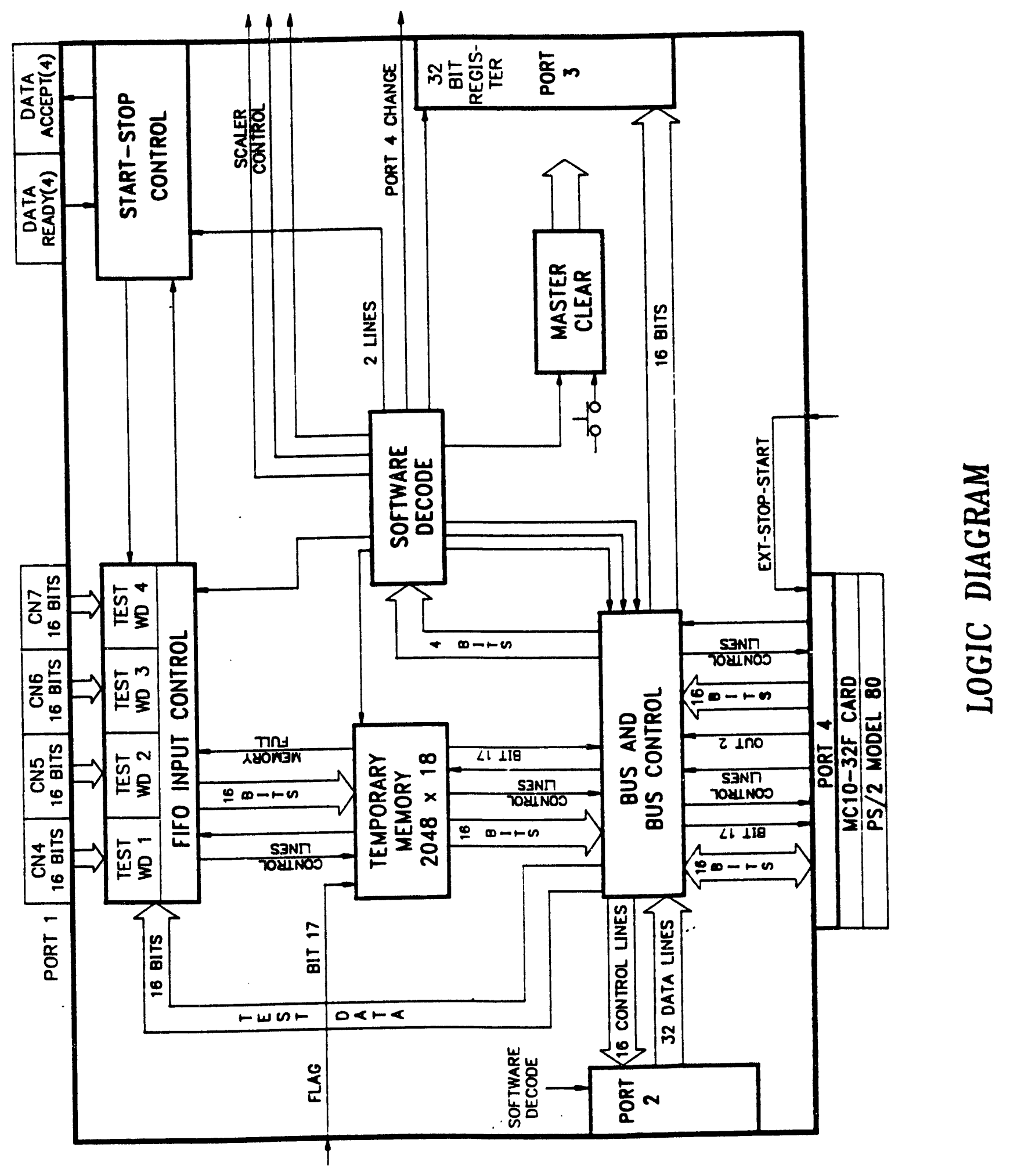




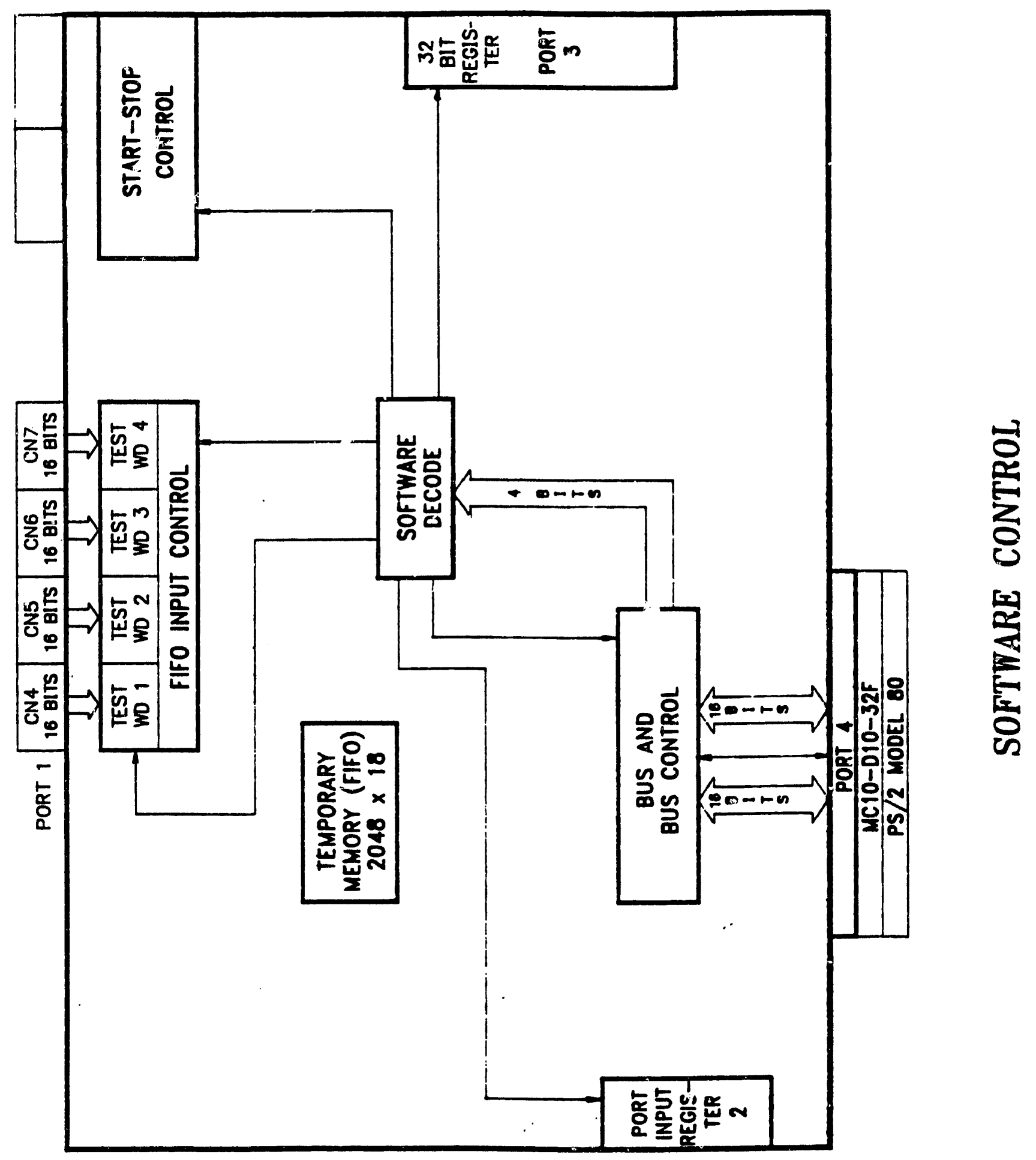




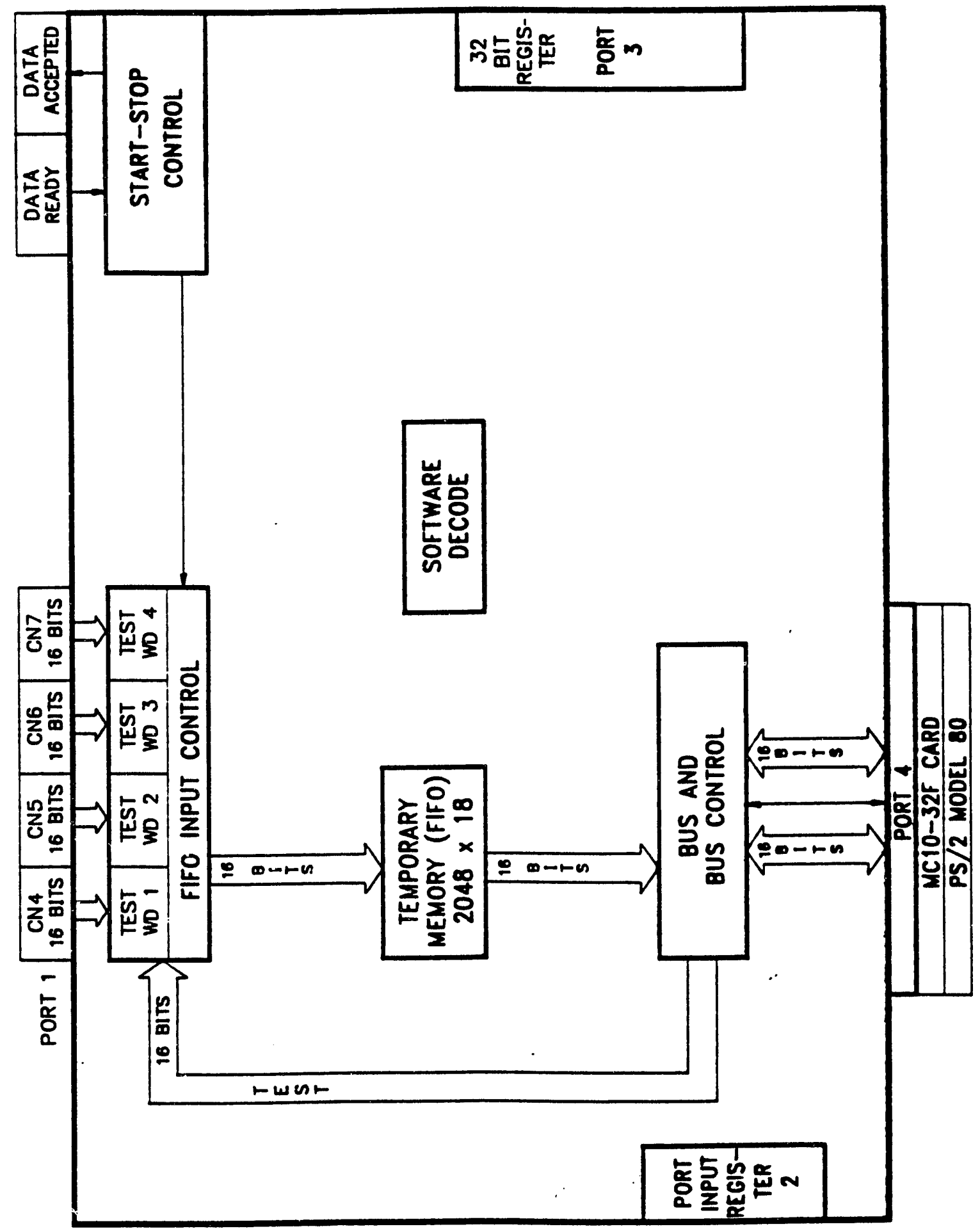

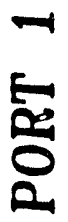




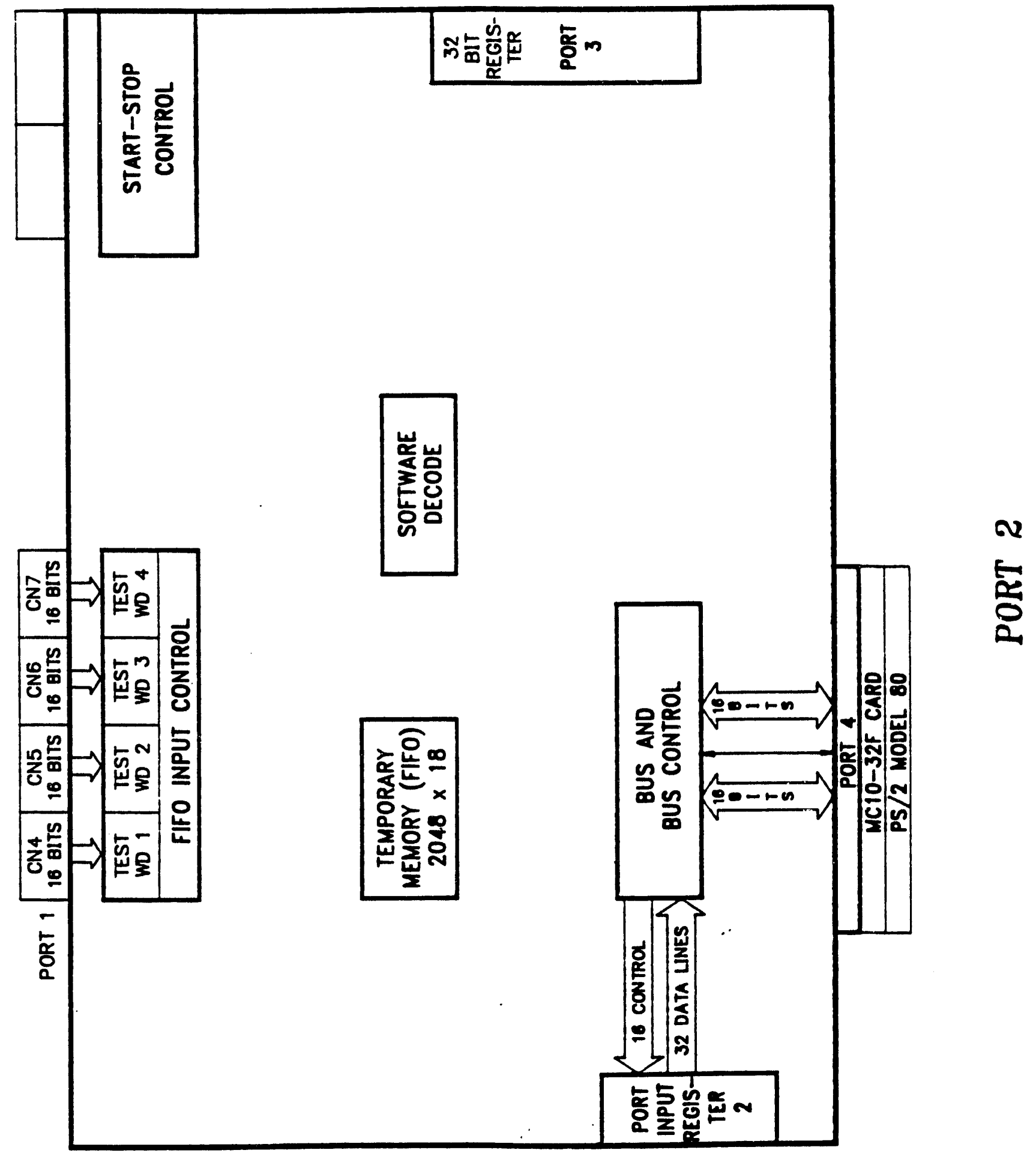




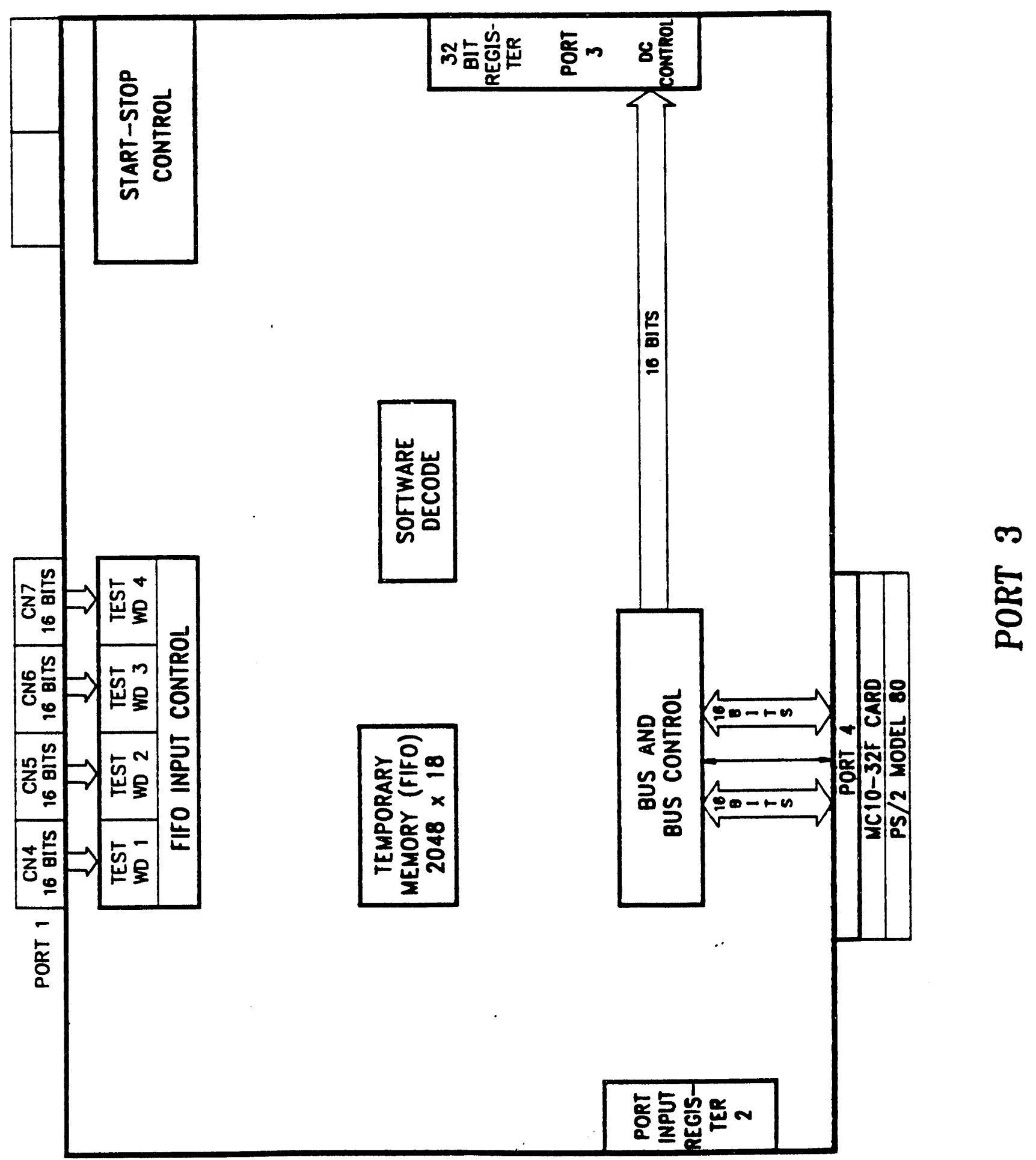




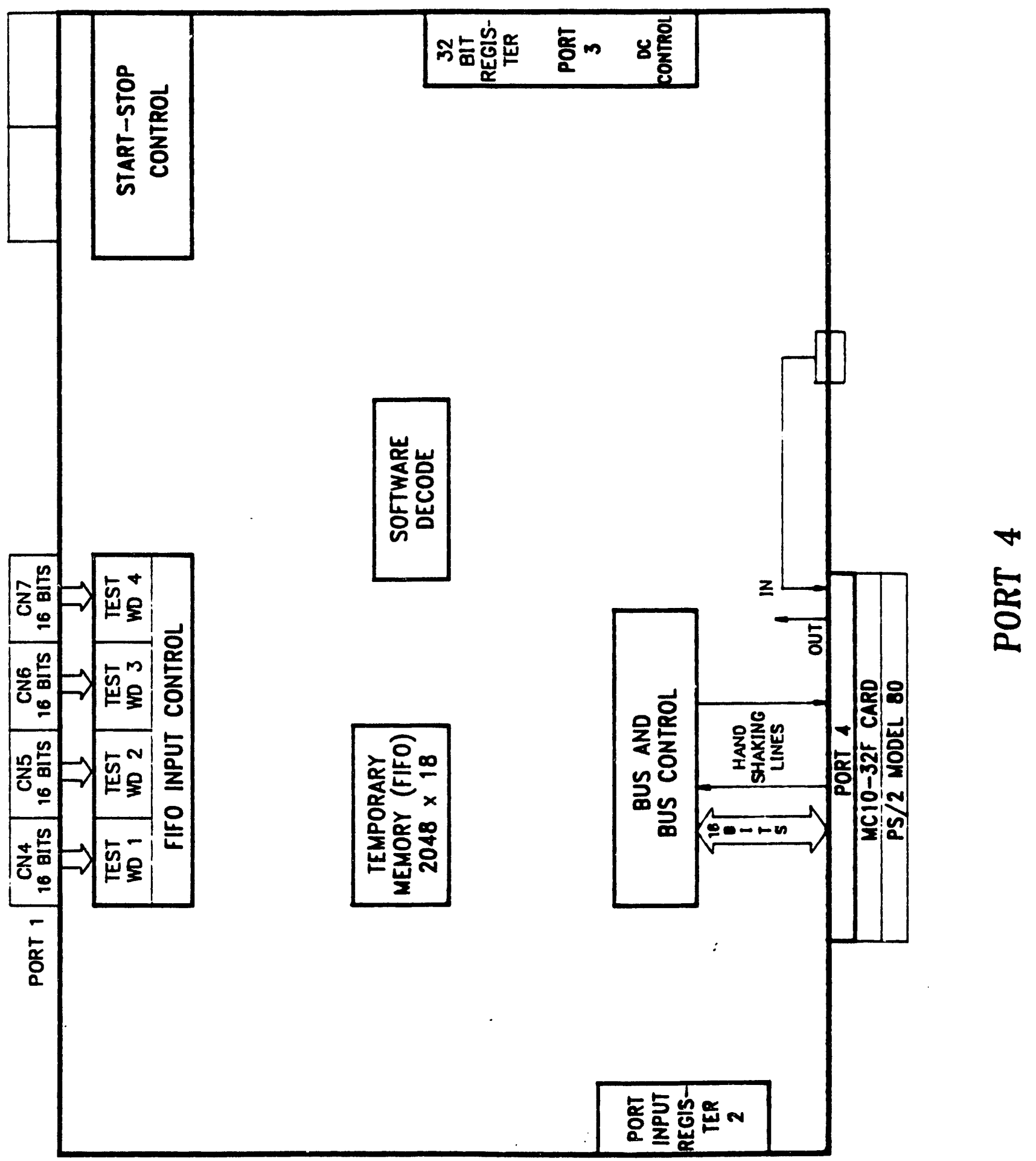




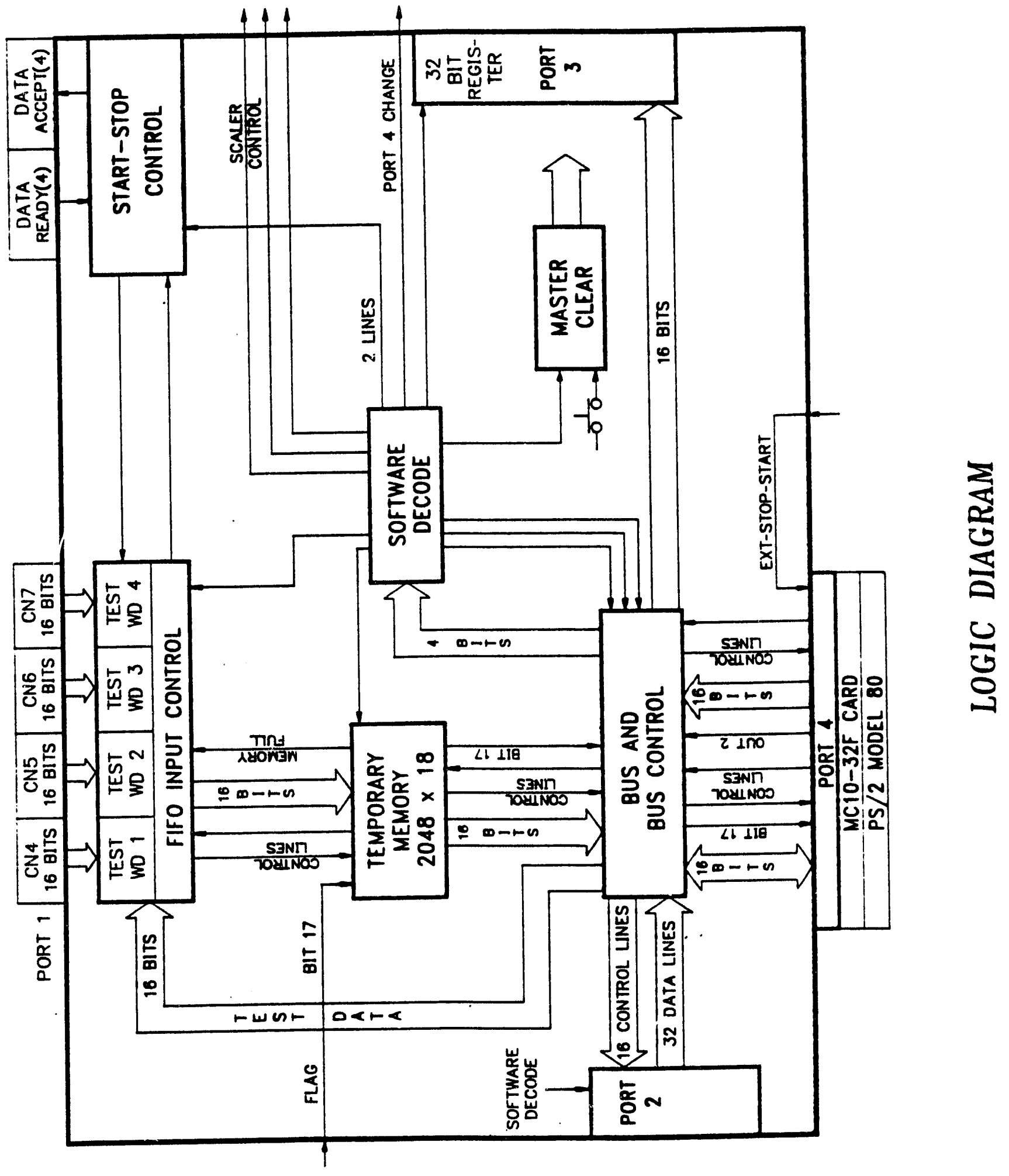



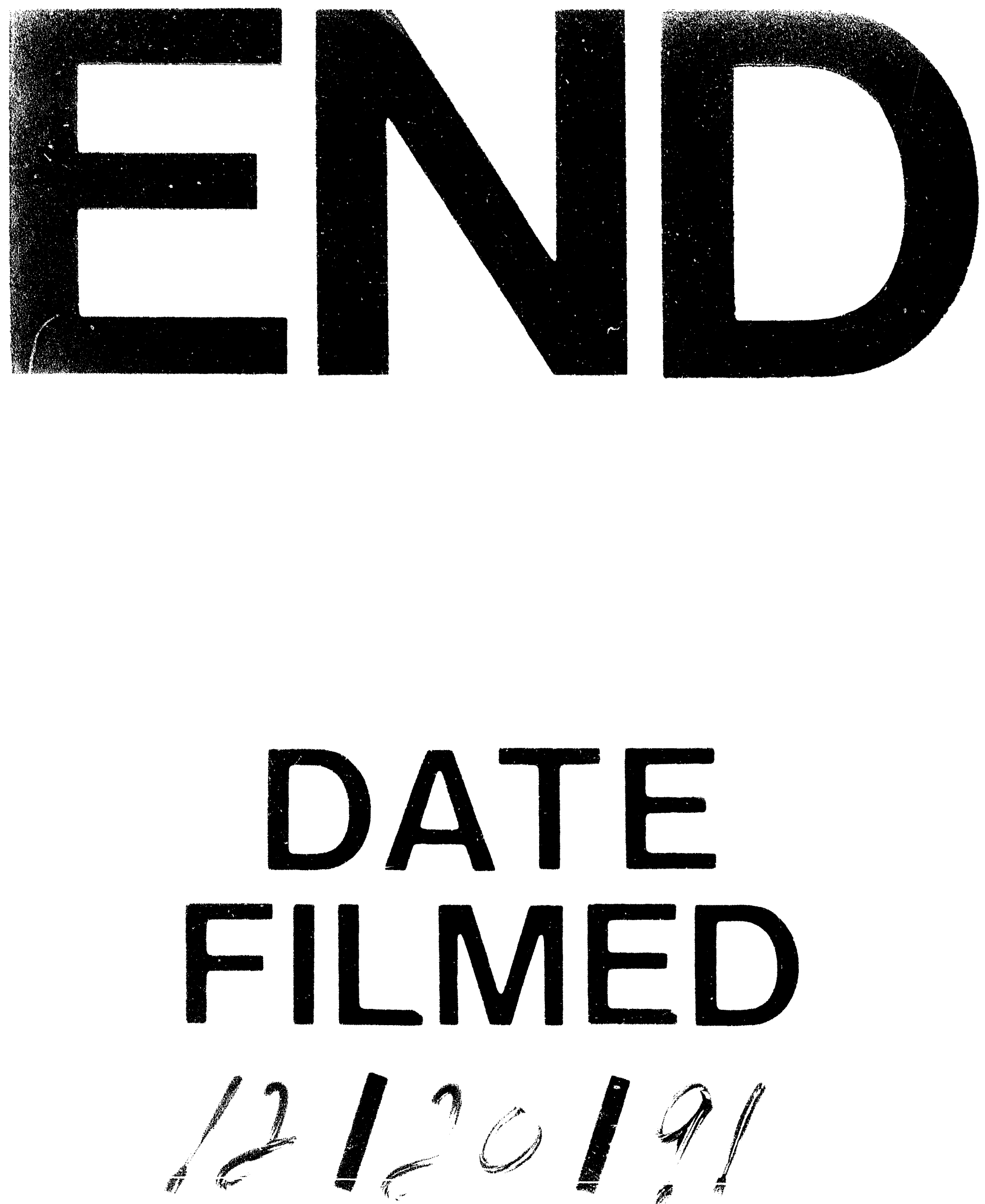
